\title{
EFFECTS OF BICARBONATE ION ON CHLOROPHYLL $a$ FLUORESCENCE TRANSIENTS AND DELAYED LIGHT EMISSION FROM MAIZE CHLOROPLASTS
}

\author{
Alan Stemler and Govindjee \\ Departments of Botany. Physiology and Biophysics, University of Illinois, Urbana. Illinois 61801 , \\ U.S.A.
}

(Received 7 June 1973; accepted 28 August 1973)

\begin{abstract}
Using high-intensity actinic light, the chlorophyll $a$ fluorescence transient from $\mathrm{HCO}_{3}{ }^{-}-$ depleted chloroplasts shows a rapid initial rise $(\mathrm{O} \rightarrow 1)$ followed by a slow phase $(I \rightarrow P)$. In the presence of $\mathrm{HCO}_{3}{ }^{-}$, the $\mathrm{O} \rightarrow \mathrm{I}$ rise is delayed but the $\mathrm{I} \rightarrow \mathrm{P}$ phase is much more rapid. Using lowintensity actinic light, the chlorophyll $a$ fluorescence transient from 3-(3.4-dichlorophenyl)-1,1 dimethylurea (DCMU)-treated chloroplasts is delayed in the presence of $\mathrm{HCO}_{3}^{-}$. Bicarbonate increases the amount of delayed light emission from chloroplasts given $10 \mathrm{~s}$ illumination with weak blue light $\left(0 \cdot 4 \mathrm{~W} / \mathrm{m}^{2}\right)$. DCMU greatly increases the amount of delayed light seen in the presence of $\mathrm{HCO}_{3}{ }^{-}$ under these conditions but decreases the amount seen in the absence of $\mathrm{HCO}_{3}^{-}$. It is suggested that $\mathrm{HCO}_{3}{ }^{-}$may somehow form or stabilize, in the dark, a number of reaction center's corresponding to the $S_{1}$ state in the model of B. Forbush, B. Kok and M. McGloin (Photochem. Photobiol. 14, 307-321, 1971).
\end{abstract}

\section{INTRODUCTION}

The ability of $\mathrm{CO}_{2}$ to stimulate the Hill reaction effected by isolated chloroplasts was first clearly demonstrated by Warburg and Krippahl (1960), and has been well documented by Izawa (1962), Good (1963), Vennesland et al. (1965), West and Hill (1967) and Stemler and Govindjee (1973), among others. It now appears that bicarbonate ion $\left(\mathrm{HCO}_{3}{ }^{-}\right)$, not dissolved $\mathrm{CO}_{2}$ gas, is the active form in this phenomenon (Stemler and Govindjee, 1973). Despite its ability to stimulate the Hill reaction, $\mathrm{HCO}_{3}{ }^{-}$was thought not to be directly involved in the primary photochemical reactions associated with oxygen evolution, as the $\mathrm{HCO}_{3}^{-}$effect was not always observed at low light intensities (lzawa, 1962; Good, 1963). Since these observations could also be explained by assuming incomplete removal of $\mathrm{HCO}_{3}{ }^{-}$, the role of this ion in photosynthetic oxygen evolution remained ambiguous. Recently, Stemler and Govindjee (1973) found that at least one site of action of $\mathrm{HCO}_{3}{ }^{-}$ occurs between the oxygen-evolving site and the electron-donation site of the artificial donor diphenyl carbazide (DPC) to photosystem II. This new evidence indicates $\mathrm{HCO}_{3}{ }^{-}$may be involved in the initial photochemical reactions leading to oxygen evolution. Further study, using maize chloroplasts, was therefore undertaken to determine the effects of $\mathrm{HCO}_{3}{ }^{-}$on chlorophyll $a(\mathrm{Chl} a)$ fluorescence transients and delayed light emission (DLE), both reflecting initial photochemical events in photosystem II.

\section{MATERIALS AND METHODS}

Maize (Zea mays) chloroplasts were isolated and depleted of bound bicarbonate in a manner already described by Stemler and Govindjee (1973). The suspension medium is described in the legends of Figs. 1, 5, and 7.

Fluorescence-transient measurements were made as described by Munday and Govindjee (1969). The photomultiplier signal was fed through a Tektronix oscilloscope (type 502) and displayed on an Esterline Angus (model E 11015 ) or a Midwestern oscillograph recorder (model $801)$. Chlorophyll $a$ fluorescence was excited with bluegreen light (Corning C.S. 4-96 plus 3-73 filters). The light intensity was $80 \mathrm{~W} / \mathrm{m}^{2}$ or lower, reduced by means of calibrated neutral-density filters. Fluorescence was observed at $685 \mathrm{~nm}$ (half band width, $6.6 \mathrm{~nm}$ ) through a Bausch \& Lomb monochromator (blazed at $750 \mathrm{~nm}$ ). A Corning C.S. 2-58 filter, placed at the entrance slit of the analyzing monochromator, eliminated stray exciting light. Recovery of the fluorescence transient in the dark was measured as the area bounded by the fluorescence transient curve after variable periods of darkness. divided by the area after a long dark period, times 100 (see Mohanty et al., 1971).

Measurements of DLE were made using the instrument described by Jursinic and Govindjee (1972). For these measurements, chloroplast suspensions were preilluminated with blue light (Corning C.S. 4-96 filter). The intensity of exciting light was $0.4 \mathrm{~W} / \mathrm{m}^{2}$ or $8.0 \mathrm{~W} / \mathrm{m}^{2}$ (in some experiments). Both DLE and fluorescence measurements were taken under anaerobic conditions. 
RESULTS AND DISCUSSION

\section{Chlorophyll a fuorescence transients}

High light intensity. Figure 1 shows the fast fuorescence transient observed in isolated chloroplasts exposed to $80 \mathrm{~W} / \mathrm{m}^{2}$ blue-green light. These traces were obtained with an Esterline Angus recorder. Careful and repeated measurements, using the faster oscillographic recorder, showed no effect of bicarbonate ions on the " $O$ " level (i.e., the constant fiuorescence; Fo). However, in bicarbonatedepleted chloroplasts, the initial fluorescence rise from the first recorded level to I (intermediate hump) fluorescence is rapid, but the $\mathbf{I} \rightarrow \mathrm{D}$ (the infiection) and the $D \rightarrow P$ (peak, $F \propto$ ) phases are slow. In the presence of $10 \mathrm{~m} M$ bicarbonate, the initial fast rise to I appears slower and is depressed, the level $D$ is not clear and occurs earlier (see insert Fig. 1), and the $\mathrm{D} \rightarrow \mathrm{P}$ rise is much more rapid with a half rise time of about $1 \mathrm{~s}$ vs $4 \mathrm{~s}$ in bicarbonate-depleted chloroplasts. The intensity of fluorescence at the $\mathrm{P}$ leveJ, like that at the $\mathrm{O}$ level, is insensitive to $\mathrm{HCO}_{3}^{-}$. In saturating light (as used here), when 3-(3,4-dichlorophenyl)-1,1 dimethylurea (DCMU) is present, the fiuorescence transient is not noticeably different with and without $\mathrm{HCO}_{3}{ }^{-}$. The rise to $P$ is very rapid, as expected, compared to the unpoisoned chloroplasts, but the extent of the $P$ level is lower by about 25 per cent in both cases.

To explain $\mathrm{Chl}$ a fluorescence transients, it is generally assumed that the yield of variable fluorescence is a function of the redox state of the primary

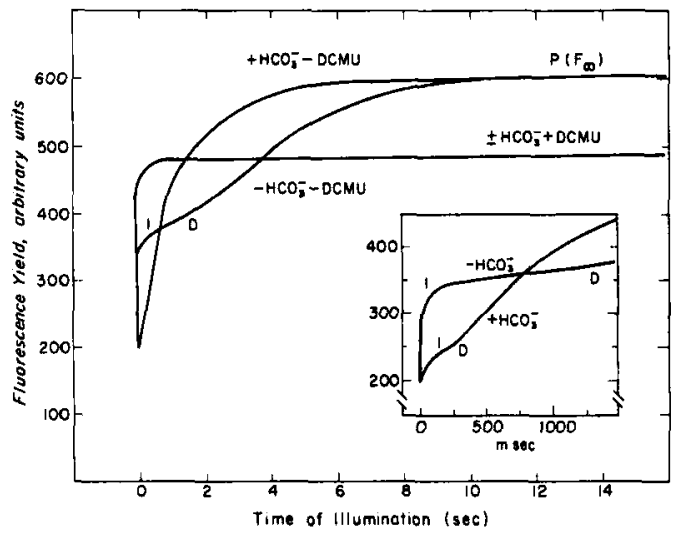

Figure 1. Fluorescence yield of chlorophyll $a$ at $685 \mathrm{~nm}$ as a function of time of high-intensity illumination in the presence and absence of $0.01 \mathrm{MNaHCO}_{3}$. Maize chloroplasts previously depleted of $\mathrm{HCO}_{3}^{-}$were suspended in $0.25 M \mathrm{NaCl}, 0.04 M \mathrm{Na}$ acetate, $0.05 M$ phosphate buffer $\mathrm{pH} 6.8, \pm 10^{-5} \mathrm{M}$ DCMU; $10 \mu \mathrm{g}$ chlorophyll $/ \mathrm{ml}$. Blue actinic light, $80 \mathrm{~W} / \mathrm{m}^{2}$. electron acceptor of photosystem II, Q (Duysens and Sweers, 1963). $\mathrm{HCO}_{3}^{-}$depletion may pose a block in electron flow either before or after $Q$. If the block occurs after $Q$, however, thus preventing the reoxidation of $Q$ by intersystem intermediates (A pool), one would expect variable fluorescence to be at all times greater in the absence of $\mathrm{HCO}_{3}{ }^{-}$. This is not the case. The I to $\mathrm{P}$ phase is instead retarded by $\mathrm{HCO}_{3}^{-}$depletion. Thus it is suggested that $\mathrm{HCO}_{3}{ }^{-}$acts prior to $\mathrm{Q}$ on the oxygen-evolving side of photosystem II, in agreement with our earlier conclusion (Stemler and Govindjee, 1973). As a working hypothesis, we suggest that $\mathrm{HCO}_{3}^{-}$may be affecting the ratio of the oxidation states $S_{0} / S_{1}$ (referring to the kinetic model and notation of Forbush et al., 1971). This ratio can be changed chemically (Bouges-Bocquet, 1973). More specifically, the $S_{0} / S_{1}$ ratio may be higher in the absence of $\mathrm{HCO}_{3}{ }^{-}$than in its presence, $\mathrm{HCO}_{3}{ }^{-}$acting to stabilize the $S_{1}$ state. The only additional assumption required is the existence of an endogenous electron donor $\mathrm{D}_{0}^{-}$(already proposed by Forbush et al., 1971 to explain dark deactivation of oxidation states $S_{2}$ and $S_{3}$ ). In the absence of $\mathrm{HCO}_{3}{ }^{-}$, the faster OI rise is explained to be due to the $S_{0} \rightarrow S_{1}$ transition and the slower IP rise to the slow donation of electrons by $\mathrm{D}_{0}^{-}$to the $S_{1}$ state, reforming the $S_{0}$, eventually reducing the $A$ pool completely. In the presence of $\mathrm{HCO}_{3}{ }^{-}$, the relativeJy smooth OP rise reflects the normal transitions from the $S_{1}$ state (stabilized by $\mathrm{HCO}_{3}^{-}$) to all higher states until the $A$ pool is likewise completely reduced. (For a detailed model, see Stemler, Ph.D. thesis, in preparation.)

If unpoisoned chloroplasts are subjected to 80 $\mathrm{W} / \mathrm{m}^{2}$ light until the $\mathrm{P}$ level is achieved, recovery of the fluorescence transient is incomplete, regardless of the dark time allowed (up to $10 \mathrm{~min}$; see Fig. 2). This may be due to the anaerobic conditions used, which would prohibit the reoxidation of electrons acceptors by oxygen or to a nonspecific effect of the high salt concentrations employed. Recovery is, however, faster and more complete in the presence of $\mathrm{HCO}_{3}{ }^{-}$. After one min of darkness, about 8.5 per cent of the transient is recovered in $\mathrm{HCO}_{3}^{-}$-depleted chloroplasts, while about 17 per cent is recovered in chloroplasts given $10 \mathrm{mM} \mathrm{HCO}_{3}{ }^{-}$; after $10 \mathrm{~min}$ dark, values are 35 and 60 per cent, respectively.

Low light intensity. Fluorescence transients were also measured at low light intensity $\left(0.4 \mathrm{~W} / \mathrm{m}^{2}\right)$ in order to allow comparison with DLE results to be reported here. At this intensity, fluorescence is much greater in the presence of DCMU (see Fig. 


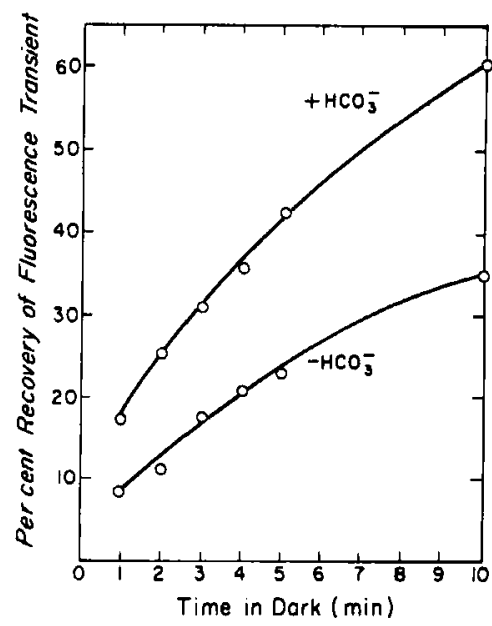

Figure 2. Recovery of the chlorophyll a fluorescence transient in the dark using unpoisoned chloroplasts. Conditions were the same as in Fig. 1.

3). $\mathrm{HCO}_{3}{ }^{-}$slows the half rise time to steady state by about 30 per cent, and lessens the extent of rise by about 15 per cent. In the absence of DCMU, very little variable fluorescence is observed although a slightly greater amount is seen in the absence of $\mathrm{HCO}_{3}{ }^{-}$.

An effect of $\mathrm{HCO}_{3}{ }^{-}$at this low light intensity and in the presence of DCMU stresses once again that $\mathrm{HCO}_{3}{ }^{-}$is affecting early photochemical events. The lower steady-state $(P)$ level of fluorescence seen in the presence of both $\mathrm{HCO}_{3}{ }^{-}$and $\mathrm{DCMU}$ may be explained by proposing a rapid back reaction (charge recombination) in the reaction centers exposed to $\mathrm{HCO}_{3}{ }^{-}$. Thus reaction centers would be 'reopened' at an appreciable rate compared to

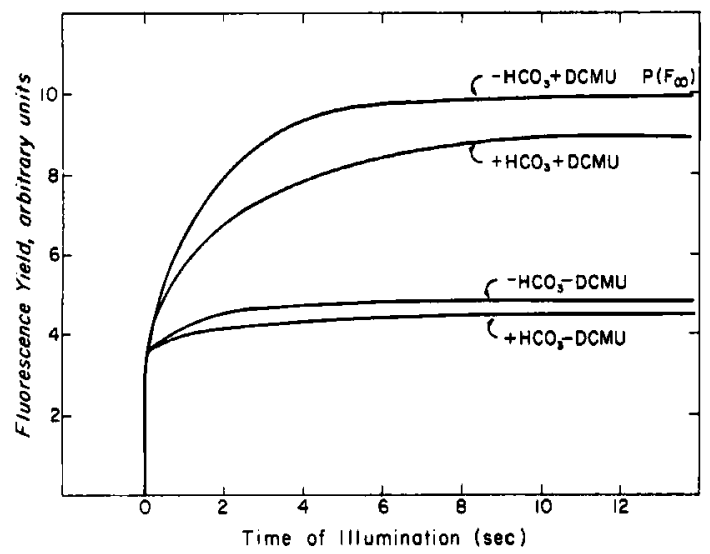

Figure 3. Fluorescence yield of chlorophyll $a$ at $685 \mathrm{~nm}$ as a function of time of low-intensity illumination. Blue actinic light, $0.4 \mathrm{~W} / \mathrm{m}^{2}$. Other conditions were the same as in Fig. 1. their rate of closing by light, suppressing the variable fluorescence. This idea is supported by both a faster rate of recovery of the fluorescence transient and a much larger amount of delayed light emission observed in the presence of both $\mathrm{HCO}_{3}{ }^{-}$and $\mathrm{DCMU}$ (vide infra).

The recovery of low-intensity fluorescence transients was measured, using DCMU-poisoned chloroplasts, with dark times up to $16 \mathrm{~s}$ only (Fig. 4). In the presence of $\mathrm{HCO}_{3}{ }^{-}$, about 14 per cent of the transient is recovered within $2 \mathrm{~s}$. Very little additional recovery is seen in the following $14 \mathrm{~s}$. In the absence of $\mathrm{HCO}_{3}{ }^{-}$, only 2-3 per cent of the transient is recovered within $2 \mathrm{~s}$. Recovery increases to about 8 per cent at $6 \mathrm{~s}$ and increases slowly to 10 per cent at $16 \mathrm{~s}$. Even after long periods of dark, recovery of the transient remains far from complete, whether or not $\mathrm{HCO}_{3}{ }^{-}$is present. After 2 min (data not shown), only about 35 per cent of the transient is recovered, and this is the same in the presence and absence of $\mathrm{HCO}_{3}{ }^{-}$. It must be pointed out that a lower steady-state $(P)$ level was used in calculating recovery (for Fig. 4) in the presence of bicarbonate than in its absence, thus reducing the area over the curve. This is significant because we propose that the lower $\mathrm{P}$ level in the presence of $\mathrm{HCO}_{3}{ }^{-}$is due to a rapid primary back reaction, i.e., charge recombination, taking place. Consequently, more photosystem II traps stay open during illumination in the presence of $\mathrm{HCO}_{3}{ }^{-}$, suppressing variable fluorescence. Since recovery was calculated on the basis of what occurred in the dark, only the per cent of photo-

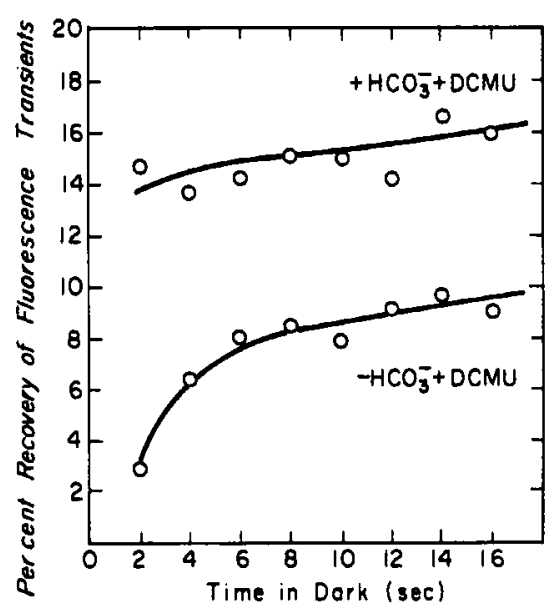

Figure 4, Recovery of the chlorophyll $a$ fluorescence transient in the dark using DCMU poisoned chloroplasts after illumination with weak blue light, $0.4 \mathrm{~W} / \mathrm{m}^{2}$. Other conditions were the same as in Fig. 1. 
synthetic units actually closed at the termination of illumination are considered in the recovery data. In other words, the per cent of traps capable of very fast recovery in the presence of $\mathrm{HCO}_{3}{ }^{-}$is probably much higher than the 14 per cent indicated in Fig. 4. In any case, we can make the qualitative conclusion that back reactions of the PS II centers are facilitated by $\mathrm{HCO}_{3}^{-}$.

\section{Delayed light emission}

Figure 5 shows the delayed light emitted, by chloroplasts, seconds after illumination for $10 \mathrm{~s}$ with $0.4 \mathrm{~W} / \mathrm{m}^{2}$ blue light. The greatest amount of delayed light is observed when chloroplasts are illuminated in the presence of both $\mathrm{HCO}_{3}^{-}$and DCMU. When DCMU is absent, the intensity of DLE is reduced by slightly more than 50 per cent. Still less (about 25 per cent of the maximum) is observed when both $\mathrm{HCO}_{3}^{-}$and $\mathrm{DCMU}$ are omitted. However, the least DLE (about 10 per cent of the maximum) is seen when $\mathrm{HCO}_{3}{ }^{-}$is omitted but DCMU is present.

When chloroplasts are illuminated for $60 \mathrm{~s}$ at low intensity $\left(0.4 \mathrm{~W} / \mathrm{m}^{2}\right)$, the pattern of DLE is different (Fig. 6). The amount of DLE is greatest in the presence of $\mathrm{HCO}_{3}^{-}$but in the absence of DCMU. A large increase (about 3-fold) is seen compared to the $10 \mathrm{~s}$ value. Relatively less (about 70 per cent of the maximum) DLE is observed

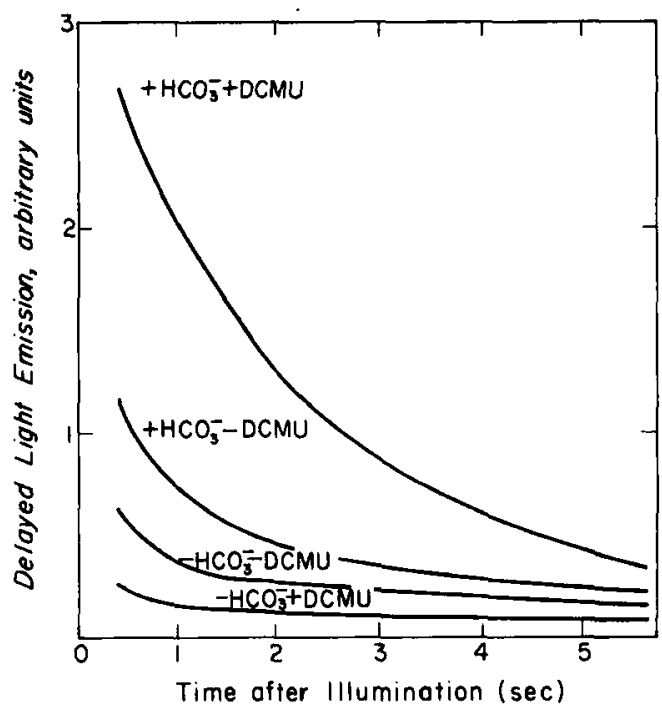

Figure 5. DLE (delayed light emission) decay from maize chloroplasts, previously depleted of $\mathrm{HCO}_{3}{ }^{-}$, illuminated for $10 \mathrm{~s}$ in weak blue light $\left(0.4 \mathrm{~W} / \mathrm{m}^{2}\right)$. Chloroplasts were suspended in $0.25 M \mathrm{NaCl}, 0.04 M \mathrm{Na}$ acetate, $0.05 M$ phosphate buffer pH 6.8, $\pm 0.01 M \mathrm{NaHCO}_{3} \pm 10^{-5} M$ DCMU. $15 \mu \mathrm{g}$ chlorophyll/ml.

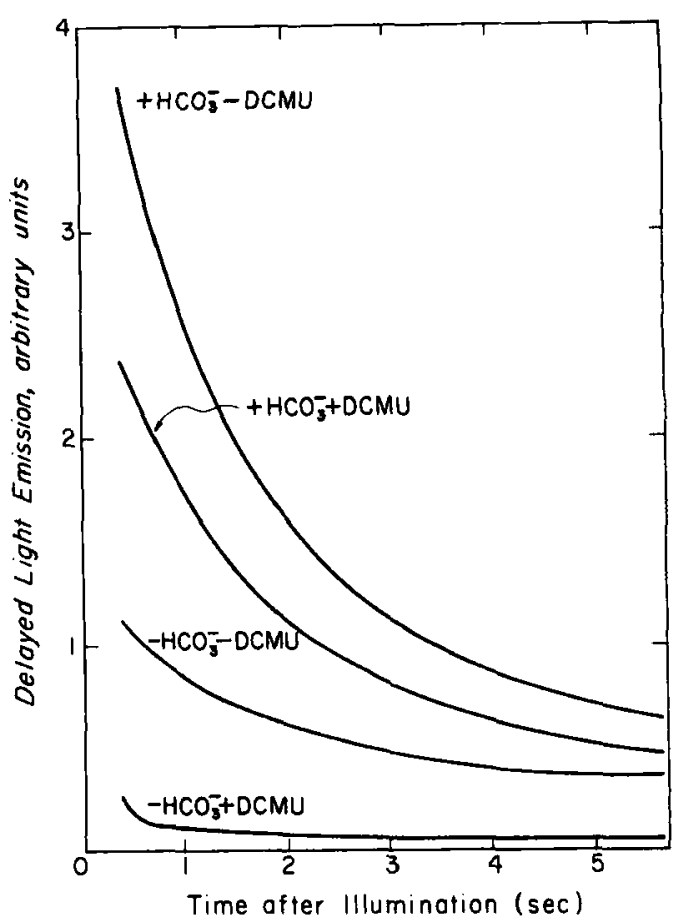

Figure 6. DLE decay from maize chloroplasts illuminated for $60 \mathrm{~s}$. All other conditions exactly as described in the legend of Fig. 5.

when $\mathrm{HCO}_{3}^{-}$and $\mathrm{DCMU}$ were both present, as this amount of DLE remained essentially unchanged from the $10 \mathrm{~s}$ value. The amount of DLE seen in the absence of both $\mathrm{HCO}_{3}{ }^{-}$and DCMU increased (about 2-fold) from the $10 \mathrm{~s}$ value but remained less (about half as much) than observed in the presence of both treatments. The least amount of DLE (about 5 per cent of maximum) is observed in the absence of $\mathrm{HCO}_{3}^{-}$but in the presence of DCMU; here an actual decline from the $10 \mathrm{~s}$ value occurs.

Short periods (10 s) of higher illumination (80 $\mathrm{W} / \mathrm{m}^{2}$, data not shown) produced results very similar to longer periods $(60 \mathrm{~s})$ of weak light (Fig. 6).

While it is obvious that $\mathrm{HCO}_{3}^{-}$is stimulating back reactions in the PS II reaction centers, the data present interesting complications. DLE is extremely low by about $10 \mathrm{~s}$ after termination of the exciting beam, yet recovery of the fluorescence transient, in DCMU-poisoned systems, is far from complete at this time in our samples. Presumably, a large population of reduced $Q$ still exists when the back reactions leading to DLE have almost ended. Perhaps an endogenous electron donor on the oxygen-evolving side of photo- 
system II is competing with $\mathrm{Q}^{-}$for $\mathrm{Z}^{+}$quite successfully and acting in the same manner as artificial electron donors such as hydroxylamine in quenching DLE (Bennoun 1970; Stacy et al., 1971, Mohanty et al., 1971).

\section{Delayed light emission induced by DCMU injection}

If DCMU is injected into a chloroplast suspension as DLE is being monitored after a 10 s illumination with weak blue light, a large increase in light emission results (Fig. 7). This effect was first observed by Clayton (1969) and confirmed by Lavorel (1973). We see, however, that $\mathrm{HCO}_{3}{ }^{-}$ must be present during illumination to observe this effect. Our new finding is that bicarbonate-depleted chloroplasts show no DLE jump upon injection of DCMU. After $60 \mathrm{~s}$ of the same illumination, a slightly different effect is seen (Fig. 8). Chloroplasts illuminated in the presence of $\mathrm{HCO}_{3}^{-}$show a less dramatic DLE jump, and $\mathrm{HCO}_{3}{ }^{-}$-depleted chloroplasts demonstrate an actual drop in DLE upon injection with DCMU.

The opposite experiment, injecting $\mathrm{HCO}_{3}{ }^{-}$while monitoring DLE from $\mathrm{HCO}_{3}{ }^{-}$-depleted chloroplasts, was also done. No effect on DLE was observed upon injection of $\mathrm{HCO}_{3}^{-}$whether or not the chloroplasts were poisoned with DCMU during illumination (data not shown). Short (10 s) periods of illumination subsequent to $\mathrm{HCO}_{3}^{-}$ injection did yield an increased DLE, however. It appears, therefore, that $\mathrm{HCO}_{3}{ }^{-}$, unlike $\mathrm{DCMU}$, must be present during illumination to effect DLE.

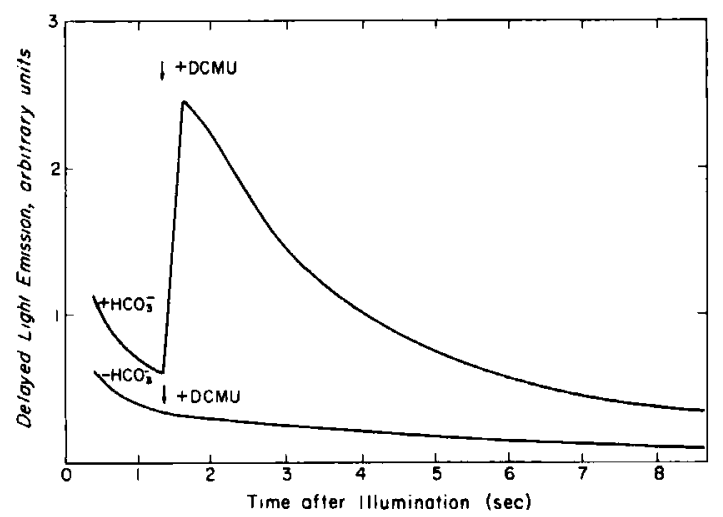

Figure 7. DLE decay from maize chloroplasts previously depleted of $\mathrm{HCO}_{3}{ }^{-}$, illuminated for $10 \mathrm{~s}$ in weak blue light $\left(0.4 \mathrm{~W} / \mathrm{m}^{2}\right), 0.2 \mathrm{ml}$ DCMU injected where indicated. Final concentrations, $0.25 M \mathrm{NaCl}, 0.04 M \mathrm{Na}$ acetate, $0.05 M$ phosphate buffer pH 6.8, $\pm 0.01 M \mathrm{NaHCO}_{3}, 15$ $\mu \mathrm{g}$ chlorophyll $\mathrm{ml}^{-1}$.

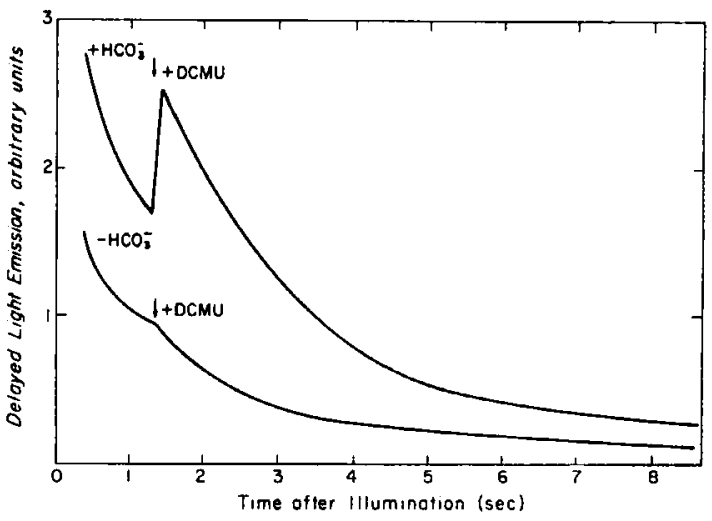

Figure 8. DLE decay from maize chloroplasts illuminated for $60 \mathrm{~s}$. All other conditions exactly as described in the legend of Fig. 7.

Clayton (1969) explained the gush of DLE seen upon injection of DCMU as simply the result of a jump in the quantum yield of fluorescence, implying that there was no increase in the rate of charge recombination per se. However this idea does not explain why there is a lack of DLE gush when DCMU is added in the absence of $\mathrm{HCO}_{3}{ }^{-}$. To explain this phenomenon, we may postulate that in the absence of $\mathrm{HCO}_{3}{ }^{-}$, the redox state of $\mathrm{Z}$ may be inappropriate for the primary back reaction to occur.

\section{CONCLUDING REMARKS}

The effects of $\mathrm{HCO}_{3}^{-}$on initial photochemical events seem in some ways contradictory. While $\mathrm{HCO}_{3}{ }^{-}$is known to stimulate oxygen evolution (Good, 1963) and ferricyanide and DCPIP reduction (West and Hill, 1961; Stemler and Govindjee, 1973), indicating greater efficiency of PS II, back reactions leading to recovery of $\mathrm{Chl} a$ fluorescence transients and DLE are also stimulated, arguing for less over-all efficiency. To render these observations compatible, we propose that $\mathrm{HCO}_{3}{ }^{-}$ permits the formation of higher oxidation states $\left(>S_{1}\right)$ of the PS II reaction centers. Such states are not only necessary for oxygen evolution, according to the kinetic model of Forbush et al. (1971) but are also the only states with a significant propensity to decay (back react) yielding much more DLE (Joliot et al., 1971; Barbieri et al., 1970). Specifically we suggest, as a working hypothesis, that $\mathrm{HCO}_{3}{ }^{-}$may do this by somehow stabilizing the $S_{1}$ state.

Whatever may be the precise role of $\mathrm{HCO}_{3}{ }^{-}$ in oxygen evolution, the fact that it markedly affects the fast $\mathrm{Chl} a$ fluorescence transient, de- 
layed light emission, reactions between the oxygenevolving site and reaction center II (Stemler and Govindjee, 1973), in addition to its possible role in photophosphorylation (Punnett and Iyer, 1964; Batra and Jagendorf, 1965), and that it even does so at low light intensities, allows the assertion that $\mathrm{HCO}_{3}{ }^{-}$is of critical importance in the initial photochemical processes of photosynthesis.

Acknowledgements-We thank the National Science Foundation for support of this work under grant GB 36751 and also the Research Board of the University of Illinois for its support.

\section{REFERENCES}

Barbieri, G., R. Delosme and P. Joliot (1970) Photochem. Photobiol. 12, 197-206.

Batra, P. and A. T. Jagendorf (1965) Plant Physiol. 40, 1074-1079.

Bennoun, P. (1970) Biochim. Biophys. Acta 216, 357-363.

Bouges-Bocquet, B. (1973) Biochim. Biophys. Acta (in press).

Clayton, R. K. (1969) Biophys. J. 9, 60-76.

Duysens, L. N. M. and H. E. Sweers (1963) In Studies on Microalgae and Photosynthetic Bacteria
(Edited by J. Ashida) pp. 353-372. University of Tokyo Press, Tokyo.

Forbush, B., B. Kok and M. McGloin (1971) Photochem. Photobiol. 14, 307-321.

Good, N. E. (1963) Plant Physiol. 38, 298-304.

Izawa, S. (1962) Plant Cell Physiol. 3, 221-227.

Jursinic, P. and Govindjee (1972) Photochem. Photobiol. 15, 331-348.

Joliot, P., A. Joliot, B. Bouges and G. Barbieri (1971) Photochem. Photobiol. 14, 287-307.

Lavorel, J. (1973) In Bioenergetics of Photosynthesis (Edited by Govindjee) Academic Press. New York (in press).

Mohanty, P., T. Mar and Govindjee (1971) Biochim. Biophys. Acta 253, 213-221.

Munday Jr., J. C. and Govindjee (1969) Biophys. J. 9.1-21.

Punnett, T. and R. V. Iyer (1964) J. Biol. Chem. 239, 2335-2359.

Stacy, W., T. Mar, Govindjee and C. Swenberg (1971) Photochem. Photobiol. 14, 197-2 19.

Stemler, A. J. and Govindjee (1973) Plant Physiol. 52, 119-123.

Vennesland. B., E. Olson and R. N. Ammeral (1965) Federation Proc. 24, 873-880.

Warburg, O. and G. Krippahl (1960) Z. Naturforsch. 156, 367-369.

West. J. and R. Hill (1967) Plant Physiol. 42, 819826. 